\title{
L'Histoire des Arts : le rêve pour apprendre
}

Rémi Azémar, Valérie De La Forest et Mary-Eve Pénancier

\section{(2) OpenEdition}

Journals

Édition électronique

URL : https://journals.openedition.org/trema/3334

DOI : $10.4000 /$ trema.3334

ISSN : 2107-0997

\section{Éditeur}

Faculté d'Éducation de l'université de Montpellier

\section{Édition imprimée}

Date de publication : 1 mai 2015

Pagination : 92 - 100

ISSN : 1167-315X

\section{Référence électronique}

Rémi Azémar, Valérie De La Forest et Mary-Eve Pénancier, «L'Histoire des Arts : le rêve pour

apprendre », Tréma [En ligne], 43 | 2015, mis en ligne le 25 juin 2015, consulté le 28 juin 2022. URL :

http://journals.openedition.org/trema/3334 ; DOI : https://doi.org/10.4000/trema.3334

Ce document a été généré automatiquement le 29 septembre 2020.

Trema 


\title{
L'Histoire des Arts : le rêve pour apprendre
}

\author{
Rémi Azémar, Valérie De La Forest et Mary-Eve Pénancier
}

\section{Introduction}

1 Bien au-delà du beau, l'ambition de l'art dans son temps a toujours été d'inspirer en ouvrant les yeux et l'esprit aux savoirs, aux idées et aux valeurs pour provoquer par les sens le dépassement de soi. Tel est le motif au cœur de l'Histoire des Arts dans les programmes de l'enseignement.

2 Comment opérer ce passage par l'émotion, par le rêve, et permettre au sensible d'enrichir la connaissance de l'élève?

3 L'Histoire des arts, mode de mise en cohérence des disciplines et des savoirs, incitation à projets, donne toute sa réalité à la continuité des apprentissages de la maternelle à la terminale. Avec la rencontre concrète des œuvres, des créateurs, des acteurs de la culture, c'est un programme d'ouverture de l'école sur la réalité sociale qui est affirmé. Mais bien au-delà, c'est un encouragement à la novation pédagogique apte à répondre à la diversité des élèves d'aujourd'hui, à leurs questions en suscitant leur sensibilité et leurs intelligences multiples dans l'action autour de l'œuvre d'art.

4 Les questions autour de cet exercice, pour les voies pédagogiques, pour les ressources et les rencontres qu'il suscite, sont posées ; à cela les chemins de traverse pour l'histoire des arts proposent les expérimentations menées par une équipe pluridisciplinaire de l'ESPE FDE - UM2 de Montpellier. Cette pratique de la formation des enseignants trouve tout son écho dans l'actualité, avec la refonte des programmes des collèges et l'accent mis sur de nouvelles pratiques pédagogiques axées autour de projets et du croisement des apprentissages disciplinaires. 


\section{L'Histoire des Arts une a-discipline}

\section{1. L'art, du singulier au pluriel} inquiétudes. Avec l'Histoire des Arts, elles ont été et sont encore nombreuses pour les enseignants. (Vieaux ${ }^{1}, 2012$ ).

6 L'invitation à l'approche sensible par l'émotion est certainement pour eux la plus grande source d'inquiétude : n'est-elle pas une mise en péril de la construction rationnelle ? Les disciplines, particulièrement les matières-pivot de l'exercice comme l'histoire et les arts visuels, ne sont-elles pas menacées dans leur identité par la fusion transdisciplinaire préconisée? Le recours à des partenariats n'annonce-t-il pas la dépossession de la «maitrise d'œuvre» pédagogique des enseignants? N'est-il pas le risque d'inégalités accrues entre les établissements avec les distorsions des offres culturelles locales?

Pour les enseignants afin d'éviter la confusion et rassurer face aux risques de dérives ou de détournements, l'emploi du pluriel contient tout dans la définition de l'Histoire des Arts qui n'est en rien de l'Histoire de l'Art, discipline clairement instituée. Cette dernière, dans sa conception classique, est tournée vers l'œuvre pour en connaître le style, les registres décoratifs, les techniques, vus avec la lecture de la création pour saisir l'intention de l'artiste. Son point fort est la dimension esthétique, pour comparer et apprécier l'importance de l'œuvre dans un classement. Si la compréhension de l'environnement social et temporel pour l'historien de l'art est nécessaire, la détermination du caractère esthétique et son jugement priment. C'est donc au final, avec l'histoire de l'art, plutôt un mouvement vers l'intérieur de l'œuvre qui s'impose là, où elle est seulement quelque chose qu'il faut savoir lire.

L'Histoire des Arts, elle, procède du principe d'une association : « l'analyse du sens, à celle des formes, des techniques et des usages» (BO 2008). Pour cet objectif, toutes les disciplines sont convoquées au travers des 6 domaines et des 5 grandes périodes. La singularité de l'exercice s'affiche donc en organisant un mouvement vers l'extérieur où l'ancrage dans l'œuvre devient une clé pour comprendre le monde dans ses multiples facettes, la variété des regards disciplinaires, et instituer des repères où chaque domaine de la connaissance se situe clairement dans ses attentes et hors de toute confusion qui puisse l'affadir, et au contraire contribue à toutes ses potentialités.

\section{2. Les chemins de la connaissance par la voie de l'émotion vers le sensible}

9 En confrontant le regard de l'élève à une œuvre d'art inscrite dans son vécu, on le conduit à user de tous ses sens. L'impression, mêlée à l'ambiance, aux couleurs et aux formes, amène simplement les mots de tous les jours pour exprimer ce qui n'appartient qu'à soi. On est là dans la puissance de la découverte, dans la force d'une rencontre où l'œuvre s'allie à la personne qui va pouvoir exprimer avec son propre langage les accents de cette fusion. Cette intercession de l'émotion est particulièrement propice à l'élève car, au-delà de l'aspect ludique que le pédagogue lui procurera, elle va se nourrir de la poésie propre à l'enfance. Cette rencontre permet de prendre au sérieux ce qui se livre au regard et à l'imaginaire pour aller vers la réflexion et entrer dans l'œuvre par une métamorphose, 
comme on entre dans le personnage au théâtre, comme on s'identifie à l'autre, différent, pour y découvrir une part de soi. L'imaginaire, avec l'étonnement, éclaire simplement ce passage par la poésie, tout en gardant la distance. Il nourrit ensuite l'analyse, avec le courage d'éclaircir l'émotion, et conduit au complexe. C'est aussi provoquer cet instant crucial, simple, qui certes n'est pas tout à fait réel, mais où l'on va pouvoir démêler la complexité du vrai. L'émotion devient le sensible, constituant de la connaissance de l'œuvre d'art pour l'élève. Il va devenir une composante de son discours pour la compréhension et un ingrédient qui contribue à la distance critique autour duquel, avec ses mots, l'élève peut construire un objet de savoir fait aussi d'intelligence sensible.

Cette approche, si elle est médiatrice de découverte, de compréhension, d'appropriation, d'entrée dans l'œuvre, sera toujours féconde pour créer et soutenir le questionnement de l'enquête afin que s'y enchaîne la construction d'une réponse qui, par un mouvement vers l'extérieur de l'œuvre, créera l'objet de connaissance et le lexique qui l'éclaire.

\section{3. L'objet d'une rencontre}

Il s'agit de faire découvrir les œuvres d'art qui entourent les élèves, leur apprendre à les ressentir, à les aimer, à les adopter (BO 2002²), à les protéger en mettant les enfants dans l'action, y compris pour expérimenter l'art. C'est pour provoquer cette rencontre avec l'art, pour faire découvrir que l'œuvre est parfois insoupçonnée, comme cette simple fontaine qui, avant d'être un point d'eau, a été un objet imaginé, pensé, réfléchi, par son concepteur, artiste ou même artisan, voire architecte ou urbaniste, cette fontaine qui est donc, avant tout, « œuvre ». C'est une vraie expérience concrète qu'il faut provoquer pour que les sens s'exercent et entrent dans cette découverte.

Pour chaque discipline la rencontre sera singulière. Pour l'historien, elle est un outil d'exception où convergent toutes les histoires, où s'enchevêtrent les relations entre les structures sociales, politiques, économiques ou religieuses. Comment pensaient-ils ? Que ressentaient-ils? C'est le chemin d'une « histoire totale » par la quête d'une parcelle de l'intention du créateur qui vibre encore, toujours active dans l'œuvre, perceptible pour qui sait la questionner, sait laisser aller son imaginaire et l'articuler avec le contexte. Pour le plasticien, le scientifique, l'apport est de même intensité. Les regards disciplinaires se complètent dans le paysage de l'œuvre, chacun éclaire ses propres plans pour saisir une totalité. Cette relation directe avec l'œuvre libère l'imagination, l'esprit créatif, l'indépendance d'esprit, l'aptitude à l'émerveillement, ce talent global où réflexion et imagination se conjuguent.

\section{L'Histoire des Arts : ressources et pratiques}

\section{1. « L'universel c'est le local moins les murs » Miguel Torga ${ }^{3}$}

13 Faut-il redire la puissance du potentiel des ressources locales inscrites dans le quotidien des élèves et la nécessité de donner du sens au lieu où ils vivent?

14 Ces ressources, bien à l'opposé d'un choix à courte vue, ouvrent à l'universel ; elles vont permettre de partager l'expérience culturelle née du contact avec l'œuvre, mais sans priver les élèves de leur identité de culture qui les distingue, pour reprendre le cœur de la 
pensée de Miguel Torga. C'est là une culture de l'authentique qui se révèle à portée de main sous toutes ses facettes.

Les ressources artistiques locales sont aussi, au-delà de l'accessibilité des supports, des liens essentiels avec les partenaires de la culture. C'est ainsi cultiver l'ancrage social d'une école partie prenante de la cité. Le texte de 2008 se place dans cette incitation qui répond pleinement à une conception ouverte de l'œuvre d'art, bien au-delà du chef d'œuvre. Cette démarche est transposable sans complexe depuis l'ordinaire jusqu'à l'exception. Ouvrir l'école au patrimoine proche et aux dynamiques partenariales locales avec les institutions culturelles, muséales et les collectivités territoriales, c'est rapprocher les apprentis citoyens des institutions et de l'idée du bien commun.

\section{2. Regards croisés}

Autour de l'Histoire des Arts, les disciplines vont se croiser, s'articuler, les «connaissances vont pouvoir se relier en compréhension» (Morin, 1999). L'Histoire des Arts permet l'articulation des disciplines par des équipes pédagogiques de projet en dépassant l'encellulement des savoirs. Le partenariat avec les institutions culturelles inclus dans les objectifs des programmes, des disciplines, des projets, crée l'accoutumance aux lieux de culture. Des convergences où chaque discipline exerce son regard singulier sur l'œuvre en envisageant ses propres temporalités, résulte l'identification des repères culturels, car aucun domaine disciplinaire ne dira jamais tout sur l'œuvre.

Pour les arts visuels par exemple, l'occasion de la rencontre avec les œuvres et l'opportunité d'un croisement entre elles amène vers la production, vers l'invention, vers le «bricolage ingénieux » (Lévi-Strauss, 1960) et sensibilise à la démarche de création. Le parcours culturel se prolongera dans la scolarité par l'émotion esthétique et l'approche sensible de l'art; les œuvres feront découvrir aux élèves la complexité du monde et l'altérité, sans les priver de l'appétence du beau.

En histoire, la relation avec l'œuvre est celle que l'historien cultive avec la trace du passé et pour lui, comme toute source, l'œuvre d'art est incapable d'autonomie, d'être une fin en soi. Ainsi il privilégie la compréhension du contexte sans attention particulière pour l'aspect et la hiérarchie esthétique de l'œuvre. Mais il est très attentif au sens du beau, au message que l'œuvre exprime et à l'empreinte de son temps. L'usage de l'art pour l'historien aiguise sa méthode critique, car les représentations qu'il donne de son époque sont parfois trompeuses, souvent le présent a décidé de la qualité des œuvres du passé, le futur c'est sûr usera d'autres arguments de reconnaissance, l'inscription de l'œuvre dans la durée et la variabilité des représentations qui l'entourent sont incontournables. Ce qui attire fondamentalement les historiens, c'est l'idée que le beau reste un concentré de son temps car l'œuvre d'art n'est pas un simple fait, il y a l'émotion qu'elle suscite encore dans le présent, avec peut-être une parcelle de celle que son créateur voulait provoquer, il va falloir concilier l'analyse avec l'approche culturelle pour répondre à cette question : "Que ressentaient-ils?». Et quel bonheur pour l'historien d'être entrainé dans le côtoiement du projet par la vision du biologiste, de l'artiste, du littéraire; ce sera source de nouvelles questions et pour eux aussi, grâce à l'imagination, qui enrichira les capacités créatrices de chaque discipline. 


\section{3. Une méthode : le parcours}

19 «Par groupes de sept ou huit, chacun accompagné d'un adulte, munis de fiches balisant un parcours de découverte transdisciplinaire, des élèves arpentent les ruelles du vieux Saint-Guilhem-le-Désert, haut lieu de l'architecture romane du Languedoc. Ils comptent ici des fenêtres, redessinent là un porche médiéval, suivent le parcours du ruisseau qui traverse le village, repèrent des formes, des matériaux, explorent l'abbatiale à la recherche d'indices, de traces, de sensations qui leur serviront plus tard en classe à répondre aux questions qui n'auront pas manqué de surgir. Telle est la méthode des parcours de Chemins de Traverse pour l'Histoire des Arts pour aider les élèves à se questionner, à structurer, à comprendre leur action, du plaisir de la découverte jusqu'aux apprentissages, en passant par la rencontre sensible d'objets patrimoniaux insérés dans leur milieu. Il y a là cet exercice d'observation, de collecte d'information, de création où art, sciences et vie de la terre, physique, etc., toutes les disciplines de l'école s'épaulent pour déchiffrer la cohérence d'un lieu et son inscription dans les temporalités. »

L'exercice expérimenté avec des élèves a également démontré toute sa pertinence avec des stagiaires ou des étudiants qui découvrent une démarche active et entrent ainsi au cœur des questions pour les futures mises en œuvre: Quelle importance donner à l'émotion? Comment passer de l'émotion au sensible? Comment entrer dans les différents dispositifs pédagogiques? Comment conjuguer les disciplines sans les altérer? Comment institutionnaliser cette démarche? Comment travailler avec les partenaires de l'école?

\section{Choix de lieux}

\section{1. Autour du Moyen Age et du fait religieux : Saint-Guilhem-le- Désert}

21 Les sites choisis pour les livrets des parcours des Chemins de Traverse, par leur caractère emblématique, leur inscription dans la temporalité et l'espace, sont la clé de la démarche. Le support du site de Saint-Guilhem-le-Désert pour l'exercice ou sa transposition à des lieux plus ordinaires est à même de démontrer que la relation concrète avec les traces et la méthode des parcours peuvent révéler leur efficacité pour des sujets complexes en faisant entrer les élèves dans la cohérence d'un lieu ici particulièrement imprégné par le fait religieux.

Le village de Saint-Guilhem-le-Désert a des potentialités idéales: l'Humanité a trouvé refuge là, dans cette montagne méditerranéenne, elle a su par ses efforts dompter les éléments, le tumulte des crues automnales et la canicule de l'été. L'implantation s'y est épanouie avec un monastère bénédictin fondé par un cousin de Charlemagne, Guillaume d'Orange, devenu Saint Guilhem. Le village devient ainsi un nœud sur les routes des pèlerinages de Saint-Jacques-de- Compostelle qui contribuent à ressouder l'Occident. Toute l'organisation du village et de son territoire est profondément marquée par l'héritage du religieux avec, de surcroît, une légende, une chanson de geste, d'abondantes sources écrites ; c'est une matière exceptionnelle pour des pans entiers des programmes qui est à la disposition de l'enquête pour que les élèves puissent découvrir la cohérence d'un lieu éclairé par toutes les disciplines. Exploration de cet espace, découverte de ses 
logiques, ressenti de ce qui en émane au travers des jeux de la lumière par exemple ; ainsi les élèves peuvent entrer dans la complexité et la singularité du fait religieux passé, grâce à cette relation étroite et sensible que facilite l'œuvre et ce qui en émane encore.

\section{2. Le village des potiers et la civilisation paysanne : Saint-Jean- de-Fos, Argileum}

Faire découvrir la civilisation paysanne qui s'est éteinte en France au milieu du XXe siècle et son étrangeté pour les élèves de la société urbaine d'aujourd'hui, tel a été l'argument des chemins de traverse, autour de la muséographie d'Argileum à Saint-Jean-de-Fos (34), consacrée à la production d'une industrie céramique destinée au quotidien jusqu'à sa disparition à la veille du 1er conflit mondial.

Là, au travers de l'objet et de ses usages, l'élève peut découvrir toutes les facettes d'un passé proche, presque présent et pourtant complètement dissipé. Il peut comprendre une mutation qui s'est accomplie dans un temps accéléré en mettant un terme à une civilisation plurimillénaire, et peut apprécier la temporalité avec sa durée et ses rythmes. C'est, avec les élèves, la construction d'un concept pour laquelle toutes les disciplines vont se conjuguer : la technologie pour les machines, les sciences et vie de la terre pour les minerais, les argiles, le bois, le feu, la chimie des mélanges d'oxydes, la transformation de la matière, les langues française et régionale pour le vocabulaire, les mathématiques pour les mesures, l'histoire pour les activités passées, la géographie pour l'évolution des paysages, l'éducation physique et sportive avec des jeux de piste pour parcourir les anciens ateliers ou les rues du village, les arts visuels pour les couleurs, les formes, les matières, et l'architecture qui s'est emparée des poteries avec les gouttières et les épis de faîtages, etc.

Et là encore s'immerger dans cette émotion qui crée le métissage, le goût pour l'altérité avec cette rencontre de l'autre passé, si différent et pourtant encore si proche.

\section{3. Tous les arts sont chez moi}

Dans leurs 6 domaines et leurs liens avec les temporalités, les arts et les œuvres vont pouvoir être saisis en tous lieux bien au-delà des chefs-d'œuvre, toujours exceptionnellement accessibles. Le monument aux morts le plus modeste, la petite église de village, les urbanismes, les objets, etc. : toutes ces traces de l'inventivité créatrice de l'Homme. C'est d'une méthode qu'il s'agit, où il faut articuler l'exercice autour d'une thématique singulière inscrite dans un contexte spatial et patrimonial particulier mais vécu par l'élève et inséré dans une logique de projet transdisciplinaire. La relation concrète avec l'authentique est la clé de voûte de l'exercice. L'articulation avec les partenariats, au-delà du soutien logistique aux projets, en est l'autre donnée forte. Les élèves entendent d'autres voix, d'autres relations aux savoirs qui renforcent la curiosité et la recherche de sens. Il y a bien sûr le cortège des incontournables autour de la construction et de la mise en œuvre des parcours où les disciplines se croisent : respecter la réglementation pour les sorties, bien s'assurer des éléments de sécurité, bien définir la posture des adultes dans les moments de découverte, structurer les moments d'enquête, les rotations des groupes si l'exercice se déroule sur une journée, apprendre aux élèves à respecter les règles de sécurité et de comportements sociaux, à respecter les consignes de travail et à suivre leur ordre, apprendre à savoir prendre son temps. Il y aura aussi tout ce 
qui relève de la recherche documentaire avec l'ensemble de ses étapes, avant et/ou après le parcours.

Cette démarche pédagogique aux ambitions civiques, formatives et culturelles se déroule dans sa complétude, animée par ses objectifs et une ambition majeure pour la connaissance et son appropriation active par les élèves.

\section{Conclusion}

L'Histoire des arts est pour l'élève un des espaces où il peut cultiver ce talent global qui allie imagination et réflexion en osant explorer tous les chemins de traverse, avec cette aptitude à s'émerveiller qui engendre l'indépendance d'esprit et l'envie de créer. L'histoire des arts est un ouvroir de pédagogie pour découvrir le monde. C'est une culture de l'expérience qui s'exerce par la force d'une rencontre avec les œuvres. L'élève, l'enfant, y discerne la complexité de ses enracinements et ainsi l'histoire des arts contribue à repousser les enfermements.

Avec la démarche des "Chemins de Traverse ", l'élève devient un artisan majeur de la construction de sa connaissance, de son savoir, il expérimente l'œuvre par le corps et l'esprit, par le faire et la pensée, par un rapport aux savoirs disciplinaires qui les unit avec la pratique de la transdisciplinarité dans la connaissance. Ce n'est ni plus ni moins que l'idéal de « l'honnête homme » de Montaigne ou de Pascal car « il est plus beau de savoir quelque chose de tout que de savoir tout d'une chose, cette universalité est la plus belle » (Pascal, «Pensées», 1670). C'est rappeler une fois de plus avec E. Morin que la réalité n'est jamais disciplinaire et qu'il faut relier les savoirs pour une connaissance qui ouvre l'esprit inventif : c'est là l'idéal de l'humaniste.

\section{BIBLIOGRAPHIE}

\section{Ouvrages}

Arasse, D., (2000). On n'y voit rien. Descriptions. Denoël, Paris.

Arbousset, G., Azémar, J., Azémar, R., Coubès, A., De la Forest, V., Ganidel, G., Hyacinthe, R., Mallet, G., Penancier, M.-E., Rambal, B. (2012), Saint-Guilhem-le-Désert, Un cahier d'activités pour découvrir les patrimoines autrement, Éd. Vallée de l'Hérault.

Arbousset G., Azémar, J., Azémar, R., Bel, J.-P., Artieres, A., De La Forest, V., Ganidel, G., Maujoin, V., Pech, R., Penancier, M.-E., Rambal, B., Vayssettes, J.-L. (à paraître). Saint-Jean-de-Fos Argileum, Un cahier d'activités pour découvrir les patrimoines autrement, Éd. Vallée de l'Hérault.

Chalumeau, Jean-Luc (1994), Les théories de l'art, Vuibert, Paris.

Duvin-Parmentier, B., (2010), Pour enseigner l'histoire des arts. Regards interdisciplinaires, Amiens SCEREN/CRDP Académie d'Amiens. 
Eco, U., (1965), L'œuvre ouverte, Trad. Chantal Roux de Bézieux avec André Boucourechliev de l'Italien, Opera aperta, 1962 Paris : Le Seuil

Levi-Strauss, C., (1962), La Pensée sauvage, Paris, Plon. (1990) Poche.

Morin, Edgar, (1999), Relier les connaissances, le défi du XXIe siècle, Seuil, Paris

Morin, Edgar, (2000), Les sept savoirs nécessaires à l'éducation du futur, Seuil, Paris

Michaux, Yves, (1993), Enseigner l'art? Analyses et réflexions sur les écoles d'art, coll. Rayon art, Nîmes.

Michaux, Yves, (1999), Critères esthétiques et jugement du goût, Éd. Jacqueline Chambon coll. Rayon art, Nîmes.

Rioux, J.-P., Sirinelli, J.-F, (1997), Pour une histoire culturelle, Seuil, Paris.

Torga, M., (1986), L'universel c'est le local moins les murs, William Blake \& $C^{\circ}$ et Barnabooth éd.

\section{Textes officiels}

Bulletin officiel n³2 du 28 août 2008.

Bulletin officiel spécial $n^{\circ} 6$ du 28 août 2008.

\section{Articles}

Pour une pédagogie du patrimoine et de l'environnement, Azémar, R., Tréma, IUFM Montpellier ${ }^{\circ}$ 20-21, avril 2003.

Comment mettre en place les premiers repères culturels à l'école avec l'histoire des arts, Azémar, R., Penancier, M.-E., 2010. CDDP 66.

Chemins de traverse, Azémar, R., Ganidel, G., De La Forest, V., Penancier, M.-É., Cahiers Pédagogiques, les arts quelle histoire ! N492 pp.28-29 2011.

\section{Sites internet}

http://www.canal-educatif.fr/art.htm?gclid=COnnzo2KjLkCFRMQtAodVRsAaQ\#

http://eduscol.education.fr/histoire-des-arts/actualites/actualites/article/les-sitesacademiques-dhistoire-des-arts.html

http://media.eduscol.education.fr/file/Programmes/59/7/

HistoireArts_Ex_miseenoeuvre_114597.pdf

\section{NOTES}

1. Christian Vieaux, Nouvel Inspecteur national des Arts Plastiques : http://www.ac- paris.fr/ portail/jcms/p1_666201/faire-la-difference-entre-histoire-de-l-art-et-histoire-des-arts? cid $=$ p1_460150\&portal=p1_460188

2. http://www.education.gouv.fr/botexte/bo020502/MENE0200882C.htm

3. "L'universel c'est le local moins les murs. C'est l'authentique qui peut être vu sous tous les angles et qui, sous tous les angles, est convaincant, comme la vérité ». Conférence donnée au Brésil en 1954. Texte paru chez William Blake \& Co. 


\section{RÉSUMÉS}

L'histoire des Arts porte un programme d'ouverture sociale et de novation pédagogique ambitieux pour l'école par la rencontre concrète et sensible avec les œuvres, celle des créateurs et des partenaires acteurs de la culture. Par la confrontation à la création, la variété des savoirs disciplinaires est là invitée à se relier pour construire une connaissance qui ouvre l'élève à l'esprit inventif, l'idéal de la culture humaniste, parfaitement inscrite dans l'actualité avec l'annonce de l'esprit de la réforme des collèges.

The history of Arts carries a program of social responsiveness and of ambitious educational novation for school by concrete and sensible meeting with the works, those of the creators and of the partners who are actors of the culture. The variety of disciplinary knowledges is invited by the confrontation to creation to connect in order to build a knowledge that opens up the creative mind of the students. It is indeed he ideal of the humanistic culture which is perfectly inherent to the recent events with the announcement of curriculum reform spirit.

\section{INDEX}

Keywords : emotion, knowledge, sensitive

Mots-clés : connaissance, émotion, sensible

\section{AUTEURS}

\section{RÉMI AZÉMAR}

Faculté d'Éducation - Université de Montpellier, ESPE-LR, EHESS, Centre d'anthropologie des sociétés rurales

\section{VALÉRIE DE LA FOREST}

Faculté d'Éducation - Université de Montpellier, ESPE-LR

\section{MARY-EVE PÉNANCIER}

Faculté d'Éducation - Université de Montpellier, ESPE-LR, LIRDEF 